Solution of the Quota Problem by a Successive-Reduction Method

Author(s): D. F. Votaw Jr.

Source: Operations Research, Vol. 6, No. 1 (Jan. - Feb., 1958), pp. 56-64

Published by: INFORMS

Stable URL: http://www.jstor.org/stable/167400

Accessed: 10/10/2011 12:29

Your use of the JSTOR archive indicates your acceptance of the Terms \& Conditions of Use, available at http://www.jstor.org/page/info/about/policies/terms.jsp

JSTOR is a not-for-profit service that helps scholars, researchers, and students discover, use, and build upon a wide range of content in a trusted digital archive. We use information technology and tools to increase productivity and facilitate new forms of scholarship. For more information about JSTOR, please contact support@ jstor.org. 


\title{
SOLUTION OF THE QUOTA PROBLEM BY A SUCCESSIVE-REDUCTION METHOD*
}

\author{
D. F. Votaw, Jr. \\ Yale University, New Haven, Connecticut
}

(Received June 10, 1957)

\begin{abstract}
This paper gives a description and illustrations of a general method of solution of the so-called 'quota problem' in personnel assignment. The introduction gives a formulation of the problem and an outline of the method of solution. In the following section, general solutions and illustrations are given for the cases of two and three job categories. An illustration for the case of five job categories is given in the concluding section. The quota problem is treated in reference $3, \mathrm{pp} .28-35$, and reference 4, pp. 15-17. The results in this paper are extensions of results in those references. The problem is similar to a special case of the personnel classification problem, reference 5 , and similar to a special case of the transportation problem, reference 1 , pp. 281-298, and reference 2 .
\end{abstract}

CONSIDER A SET of persons and a set of job categories, and suppose that for each job category there is a quota. Suppose further that for each person it is known with regard to each job category whether he is 'qualified' or 'not qualified.' The quota problem can be stated as follows: (a) Does there exist an assignment of persons to jobs such that each person is qualified on the job to which he is assigned? (b) If the answer to (a) is yes, find such an assignment.

Let $n$ be the number of job categories and let the $n$ quotas be represented by $b_{1}, \cdots, b_{n}$. The answer to part $(a)$ of the quota problem is yes or no according as the following set of $2^{n}-1$ inequalities is or is not satisfied:

$$
\begin{gathered}
g_{1} \geqq b_{1}, \quad g_{2} \geqq b_{2}, \quad \cdots, \quad g_{n} \geqq b_{n}, \\
g_{12} \geqq b_{1}+b_{2}, \quad \cdots, \quad g_{n-1} \geqq b_{n-1}+b_{n}, \\
\ldots . \quad \ldots \quad c \quad \cdots \\
g_{12 \cdots n} \geqq b_{1}+b_{2}+\cdots+b_{n},
\end{gathered}
$$

where $g_{h_{1} \ldots h_{r}}$ represents the number of persons qualified in at least one of the job categories $h_{1}, \cdots, h_{r}(r \leqq n)$.

* This research was supported in part by the United States Air Force under Contract No. AF 41(657)-4, monitored by the Personnel Laboratory, Air Force Personnel and Training Research Center, P. O. Box 1557, Lackland Air Force Base, Texas. Permission is granted for reproduction, translation, publication, and use, in whole and in part, by or for the United States Government. 
Before dealing directly with part $(b)$ of the quota problem we shall introduce further notation. It is interesting that there are at most $2^{n}$ categories of persons relative to the $n$ job categories. This follows since $2^{n}$ is the total number of distinct sequences of $n$ labels when each label is one of two alternatives ('qualified,' 'not qualified'). We shall represent any of the $2^{n}$ personnel categories by $\left(i_{1}, \cdots, i_{n}\right)$ where $i_{j}=1$ or 0 according as the persons in that personnel category are or are not qualified in the $j$ th job category $(j=1, \cdots, n)$. Let $a_{i_{1} \cdots i_{n}}$ be the number* of persons in the personnel category $\left(i_{1}, \cdots, i_{n}\right)$. We shall term $a_{i_{1} \cdots i_{n}}$ the 'size' of the personnel category $\left(i_{1}, \cdots, i_{n}\right)$. The $a$ 's and the $g$ 's are related; for example, when $n=2$, we have [see (1)]

$$
g_{1}=a_{10}+a_{11}, \quad g_{2}=a_{01}+a_{11}, \quad g_{12}=a_{10}+a_{01}+a_{11} .
$$

Let $\left\|x_{i_{1} \ldots i_{n} ; j}\right\|$ be an 'allocation matrix'; any element $x_{i_{1} \ldots i_{n} ; j}$ represents a possible number of persons in personnel category $\left(i_{1}, i_{2}, \cdots, i_{n}\right)$ allocated to jobs in job category $j$. This matrix is of order $2^{n} \times n$. It should be remarked at the outset that

$$
\text { every } \quad \begin{aligned}
& x_{i_{1} \cdots i_{n} ; j} \geqq 0, \\
& \\
& x_{i_{1} \cdots i_{n} ; j}=0 \text { when } i_{j}=0 .
\end{aligned}
$$

When the answer to part ( $a$ ) of the quota problem is yes, there exists an allocation matrix $\left\|x_{i_{1} \ldots i_{n} ; j}\right\|$ such that

$$
\begin{array}{lr}
\sum_{j=1}^{j=n} x_{i_{1} \cdots i_{n} ; j} \leqq a_{i_{1} i_{2} \ldots i_{n}}, & \text { (for each set } i_{1}, i_{2}, \cdots, i_{n} \text { ) } \\
\sum x_{i_{1} \ldots i_{n} j}=b_{j}, & \text { (for each } j=1, \cdots, n \text { ) }
\end{array}
$$

where the summation in the second line of (4) is over all $2^{n}$ personnel categories $\left(i_{1}, i_{2}, \cdots, i_{n}\right)$. Given that the answer to part $(a)$ is yes, the set of all solutions of (4) is the set of all solutions of part $(b)$.

To begin the successive-reduction method we choose an $x_{i_{1} \ldots i_{n} ; j}$ that can be made positive without reversing any of the inequalities that correspond to those in (1). By "inequalities that correspond to those in (1)" we mean inequalities obtained when $a_{i_{1} \ldots i_{n}}$ is replaced by $a_{i_{1} \ldots i_{n}}-x_{i_{1} \ldots i_{n} ; j}$ and $b_{j}$ is replaced by $b_{j}-x_{i_{1} \ldots i_{n} ; j}$. Incidentally, it is always possible to choose such an $x_{i_{1} \ldots i_{n} ; j}$ when the answer to part $(a)$ is yes, since the number of $x$ 's is finite. We then set $x_{i_{1} \ldots i_{n} ; j}$ equal to the largest possible value that it may assume. This reduces the original problem to one or more smaller order problems since $x_{i_{1} \ldots i_{n} ; j}$ takes the value $a_{i_{1} \ldots i_{n}}$, or it takes the value $b_{j}$, or an equality appears among the inequalities that correspond to

* Throughout this paper we shall regard the $a$ 's and $b$ 's as integers. The results can be extended readily without any essential change in wording to include population proportions. 
those in (1) (other than the one in the last line). This completes the first step of the method.

When an equality is produced, the total of the (reduced) quotas of what ever job categories are included equals the total of persons who are qualified in at least one of those categories. All those persons must be used just to fill those quotas. Accordingly, we separate that group of job categories from the other job categories and separate the corresponding group of personnel categories from the other personnel categories. The original problem is thereby replaced by two or more quota problems of smaller order.

Having completed the first step of the method, we repeat the reduction procedure on the reduced problem or problems obtained in the first step. Continuing, we ultimately reduce the original problem to quota problems involving not more than three job categories, which can be solved quickly. General solutions of problems having not more than three job categories are given in the next section. In the final section a solution of a problem having five job categories is obtained by the successive-reduction method.

We shall now show that the quota problem resembles special cases of the personnel classification problem and the transportation problem. As regards personnel classification let us suppose that the productivity of any person on any job is expressed simply as 0 or 1 (see reference 5 , pp. 255-258). Part (a) of the quota problem can be interpreted here to mean: Is there an assignment of persons to jobs such that the total production is $b_{1}+b_{2}+\cdots+b_{n}$ ? Of course, part $(b)$ of the problem is: If the answer to $(a)$ is yes, find such an assignment. Incidentally, the quota problem is formulated in reference 5, p. 258. Turning now to transportation, let personnel categories correspond to origins, job categories correspond to destinations, and productivities correspond to savings (negative costs). Thus there are $2^{n}$ origins and $n$ destinations; the amount at origin $\left(i_{1}, \cdots, i_{n}\right)$ is $a_{i_{1} \cdots i_{n}}$ and the amount required at destination $j$ is $b_{j}$. Each saving is 0 or 1 . Part $(a)$ of the quota problem corresponds to the following question: Is there a shipping schedule such that all amounts required at destinations are supplied and every origin-destination route used involves a saving of 1 ? Part $(b)$ is: If the answer to $(a)$ is yes, find such a shipping schedule.

In view of its similarity to the personnel classification and transportation problems, the quota problem could be solved by means of the simplex method (see references 2 and 5). In general, however, the successivereduction method requires less time and effort than the simplex method. The successive-reduction method has been programmed on an electronic computer for all problems in which the number of job categories is 8 or less. 


\section{SOLUTION OF THE QUOTA PROBLEM WHEN THE NUMBER OF JOB CATEGORIES IS TWO OR THREE}

For THE case $n=2$, the matrix $\left\|x_{\imath_{1} \cdots i_{n} ; j}\right\|$ can be written as follows:

$$
\left\|\begin{array}{cc}
x_{00 ; 1} & x_{00 ; 2} \\
x_{10 ; 1} & x_{10 ; 2} \\
x_{01 ; 1} & x_{01 ; 2} \\
x_{11 ; 1} & x_{11 ; 2}
\end{array}\right\| .
$$

In this case there are two quotas, $b_{1}, b_{2}$ and four personnel category sizes, namely $a_{00}, a_{10}, a_{01}, a_{11}$. When the answer to part $(a)$ is yes, the following values of the elements of (5) provide a solution of the problem:

$$
\begin{array}{ll}
x_{00 ; 1}=0 & x_{01 ; 1}=0 \\
x_{00 ; 2}=0 & x_{01 ; 2}=\min \left(b_{2}, a_{01}\right) \\
x_{10 ; 1}=\min \left(b_{1}, a_{10}\right) & x_{11 ; 1}=b_{1}-\min \left(b_{1}, a_{10}\right) \\
x_{10 ; 2}=0 & x_{11 ; 2}=b_{2}-\min \left(b_{2}, a_{01}\right),
\end{array}
$$

where 'min' means 'minimum of.' The values of $x_{10 ; 1}$ and $x_{01 ; 2}$ in (6) were obtained by using the first step of the successive-reduction method twice. The values of $x_{11 ; 1}$ and $x_{11 ; 2}$ were then obtained by subtraction from the respective quotas.

Assume that in a quota problem involving exactly 2 job categories $b_{1}=50$ and $b_{2}=50$ and the personnel category sizes are $a_{00}=5, a_{10}=60$, $a_{01}=20, a_{11}=40$. Here we have 100 jobs to be filled and a total of 125 persons to fill them. Let us first note whether it is possible to fill the quotas with qualified persons. From the given information we have that

$$
\begin{aligned}
g_{1} & =a_{10}+a_{11}=100 \geqq b_{1}=50 \\
g_{2} & =a_{01}+a_{11}=60 \geqq b_{2}=50 \\
g_{12} & =a_{10}+a_{01}+a_{11}=120 \geqq b_{1}+b_{2}=100 .
\end{aligned}
$$

In view of (7) the answer to part $(a)$ is yes [see (1)]. To solve part $(b)$ we use (6) and obtain the following results:

$$
\begin{array}{ll}
x_{00 ; 1}=0 & x_{01 ; 1}=0 \\
x_{00 ; 2}=0 & x_{01 ; 2}=20 \\
x_{10 ; 1}=50 & x_{11 ; 1}=50-50=0 \\
x_{10 ; 2}=0 & x_{11 ; 2}=50-20=30 .
\end{array}
$$


This solution can be conveniently presented in matrix form, as follows:

$$
\left\|x_{i_{1} i_{2} ; j}\right\|=\| \begin{array}{rr}
0 & 0 \\
50 & 0 \\
0 & 20 \\
0 & 30
\end{array} \mid \begin{aligned}
& a_{00}=5 \\
& a_{10}=60 \\
& a_{01}=20 \\
& a_{11}=40
\end{aligned}
$$

Quotas: $\quad 50 \quad 50$

It should be noticed that the sum of the numbers in a column equals the quota associated with the column, and the sum of the numbers in a row does not exceed the personnel category size associated with the row.

The set of all solutions of the problem when part $(a)$ is satisfied is indicated below [see the remarks below (4)]:

$$
\begin{gathered}
x_{10 ; 1}=b_{1}-x_{11 ; 1}, \quad x_{01 ; 2}=b_{2}-x_{11 ; 2}, \\
b_{1}-a_{10} \leqq x_{11 ; 1} \leqq b_{1}, \quad b_{2}-a_{01} \leqq x_{11 ; 2} \leqq b_{2}, \\
x_{11 ; 1}+x_{11 ; 2} \leqq a_{11}, \quad x_{11 ; 1} \geqq 0, \quad x_{11 ; 2} \geqq 0 .
\end{gathered}
$$

In the particular numerical example treated above the set of all solutions is as follows:

$$
\begin{gathered}
x_{01 ; 1}=50-x_{11 ; 1}, \quad x_{01 ; 2}=50-x_{11 ; 2}, \\
0 \leqq x_{11 ; 1} \leqq 50, \quad 30 \leqq x_{11 ; 2} \leqq 50, \quad x_{11 ; 1}+x_{11 ; 2} \leqq 40 .
\end{gathered}
$$

A general solution of the quota problem when there are exactly three job categories will now be given. Here $n=3$, and there are $2^{3}=8$ personnel categories. The allocation matrix $\left\|x_{i_{1} i_{2} i_{3} ; j}\right\|$ is of order $8 \times 3$. The following matrix is a solution of part $(b)$ of the quota problem when, of course, the answer to part $(a)$ is yes:

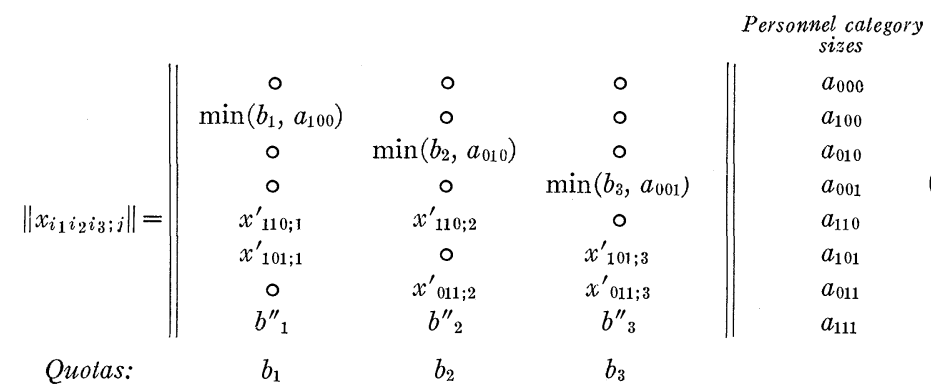

The new symbols in (12) are defined as follows:

$$
\begin{aligned}
& x_{110 ; 1}^{\prime}=\min \left(b^{\prime}{ }_{1}, a_{110}, a_{110}+a_{011}+a_{111}-b^{\prime}{ }_{2}\right) \\
& x_{110 ; 2}^{\prime}=\min \left(b^{\prime}{ }_{2}, a_{110}-x^{\prime}{ }_{110 ; 1}, a_{110}+a_{101}+a_{111}-b^{\prime}{ }_{1}\right) \\
& x_{101 ; 1}=\min \left(b^{\prime}{ }_{1}-x^{\prime}{ }_{110 ; 1}, a_{101}, a_{101}+a_{011}+a_{111}-b^{\prime}{ }_{3}\right)
\end{aligned}
$$




$$
\begin{aligned}
& x^{\prime}{ }_{101 ; 3}=\min \left(b^{\prime}{ }_{3}, a_{101}-x^{\prime}{ }_{101 ; 1}, a_{110}-x_{110 ; 2}^{\prime}+a_{101}+a_{111}-b_{1}^{\prime}\right) \\
& x^{\prime}{ }_{011 ; 2}=\min \left(b_{2}^{\prime}-x^{\prime}{ }_{110 ; 2}, a_{011}, a_{101}-x^{\prime}{ }_{101 ; 1}+a_{011}+a_{111}-b^{\prime}{ }_{3}\right) \\
& x^{\prime}{ }_{011 ; 3}=\min \left(b^{\prime}{ }_{3}-x^{\prime}{ }_{101 ; 3}, a_{011}-x^{\prime}{ }_{011 ; 2}, a_{110}-x^{\prime}{ }_{110 ; 1}+a_{011}+a_{111}-b^{\prime}{ }_{2}\right) \\
& b^{\prime}{ }_{1}=b_{1}-\min \left(b_{1}, a_{100}\right), \quad b^{\prime}{ }_{2}=b_{2}-\min \left(b_{2}, a_{010}\right), \quad b^{\prime}{ }_{3}=b_{3}-\min \left(b_{3}, a_{001}\right), \\
& b^{\prime \prime}{ }_{1}=b^{\prime}{ }_{1}-x^{\prime}{ }_{110 ; 1}-x^{\prime}{ }_{101 ; 1}, \quad b^{\prime \prime}{ }_{2}=b^{\prime}{ }_{2}-x^{\prime}{ }_{110 ; 2}-x^{\prime}{ }_{011 ; 2}, \quad b^{\prime \prime}{ }_{3}=b^{\prime}{ }_{3}-x^{\prime}{ }_{101 ; 3}-x^{\prime}{ }_{011 ; 3} \text {. }
\end{aligned}
$$

The entries in (12) were obtained by using steps of the successive-reduction method described earlier. The entries were chosen as follows: first, those in the second, third, and fourth rows of $(12)$; then $x^{\prime}{ }_{110 ; 1}, \cdots, x^{\prime}{ }_{011 ; 3}$ in the order listed in (13); and, finally, $b^{\prime \prime}{ }_{1}, b^{\prime \prime}{ }_{2}$, and $b^{\prime \prime}{ }_{3}$, which were chosen by subtraction from the quotas. The minima used in expressing $x_{110 ; 1}^{\prime}, \cdots, x_{011 ; 3}^{\prime}$ indicate the possible occurrence of equalities mentioned in the description of the successive-reduction method.

A numerical illustration will now be given for the case in which there are three job categories. Suppose the quotas and personnel category sizes are as follows:

$$
\begin{aligned}
& b_{1}=30, \quad b_{2}=30, \quad b_{3}=40, \\
& a_{000}=10, \quad a_{100}=10, \quad a_{010}=10, \quad a_{001}=15 \text {, } \\
& a_{110}=20, \quad a_{101}=20, \quad a_{011}=20, \quad a_{111}=5 \text {. }
\end{aligned}
$$

In this example the total number, say $N$, of persons available for assignment is $a_{000}+a_{100}+\cdots+a_{111}=110$. We first check as to whether there exists a solution-i.e., as to whether the answer to part $(a)$ is yes. To do this we evaluate the $g$ 's and use (1) as follows:

$$
\begin{aligned}
g & \\
g_{1} & =a_{100}+a_{110}+a_{101}+a_{111}=55 \\
g_{2} & =a_{010}+a_{110}+a_{011}+a_{111}=70 \\
g_{3} & =a_{001}+a_{101}+a_{011}+a_{111}=60 \\
g_{12} & =N-a_{000}-a_{001}=85 \\
g_{13} & =N-a_{000}-a_{010}=90 \\
g_{23} & =N-a_{000}-a_{100}=90 \\
g_{123} & =N-a_{000}=100
\end{aligned}
$$

Sum of b's

$b_{1}=30$

$b_{2}=30$

$b_{3}=40$

$b_{1}+b_{2}=60$

$b_{1}+b_{3}=70$

$b_{2}+b_{3}=70$

$b_{1}+b_{2}+b_{3}=100$

Each $g$ in (14) is not less than the corresponding sum of $b$ 's; therefore the answer to part $(a)$ is yes. To find a solution-i.e., to solve part (b)-we use (12) and obtain the following matrix:

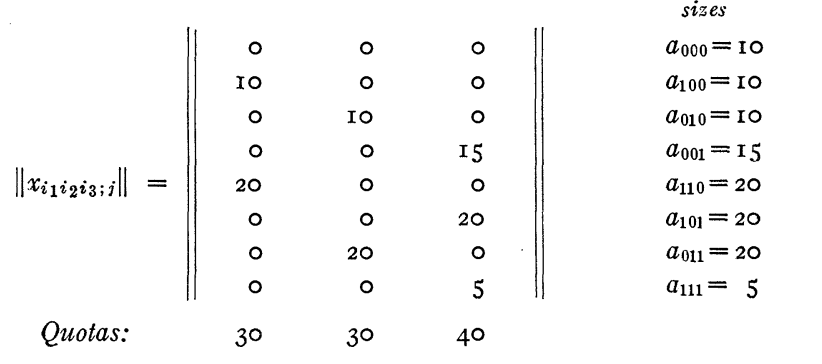


It is easily verified that (15) is a solution since each entry is a nonnegative integer, the sum of elements in a row is not greater than the corresponding personnel category size, and the sum of elements in a column equals the corresponding quota.

\section{ILLUSTRATION OF THE SOLUTION WHEN THERE ARE FIVE JOB CATEGORIES}

A NUMERICAL illustration of the successive-reduction method will now be given for the case of five job categories. Let the quotas be $b_{1}=25,000$, $b_{2}=5,000, b_{3}=35,000, b_{4}=32,500, b_{5}=2,500$. There are 32 personnel categories when $n=5$. Suppose the personnel category sizes are as those given below:

\begin{tabular}{|c|c|c|c|c|c|}
\hline$a_{00000}=1$ & {$[1, \infty 00$} & $a_{01010}=$ & $2, \infty 00$ & $a_{01110}=$ & $3, \infty 00$ \\
\hline$a_{10000}=$ & $I, 500$ & $a_{01001}=$ & $5, \infty 00$ & $a_{01101}=$ & $3, \infty 00$ \\
\hline$a_{01000}=$ & I , 500 & $a_{00110}=$ & $I, 500$ & $a_{01011}=$ & $3, \infty 00$ \\
\hline$a_{00100}=$ & $I, 500$ & $a_{00101}=$ & $3, \infty 00$ & $a_{00111}=$ & $5, \infty$ \\
\hline$a_{00010}=$ & $x, 500$ & $a_{00011}=$ & $2, \infty 00$ & $a_{11110}=$ & $5, \infty 00$ \\
\hline$a_{00001}=$ & I, 500 & $a_{11100}=$ & I, 500 & $a_{11101}=$ & $5, \infty 00$ \\
\hline$a_{11000}=$ & $2, \infty 00$ & $a_{11010}=$ & I , 500 & $a_{11011}=$ & $7, \infty$ \\
\hline$a_{10100}=$ & $2, \infty 00$ & $a_{11001}=$ & $2, \infty 00$ & $a_{10111}=$ & $7, \infty 00$ \\
\hline$a_{10010}=$ & $2, \infty 00$ & $a_{10110}=$ & $3, \infty 00$ & $a_{01111}=$ & $7, \infty$ \\
\hline$a_{10001}=$ & $3, \infty 00$ & $a_{10101}=$ & $3, \infty 00$ & $a_{11111}=$ & $10, \infty$ \\
\hline$a_{01100}=$ & $2, \infty 00$ & $a_{10011}=$ & 3,000 & & \\
\hline
\end{tabular}

In this example the total number of persons required is 100,000 $\left(=b_{1}+\cdots+b_{5}\right)$ and the total number, $N$, of persons available for assignment is 112,000 ( $\left.=a_{00000}+\cdots+a_{11111}\right)$. We first check as to whether the answer to part $(a)$ is yes. To do this we evaluate the $g$ 's and compare each with the corresponding sum of $b$ 's [see (1)]. The comparisons can be made as follows:

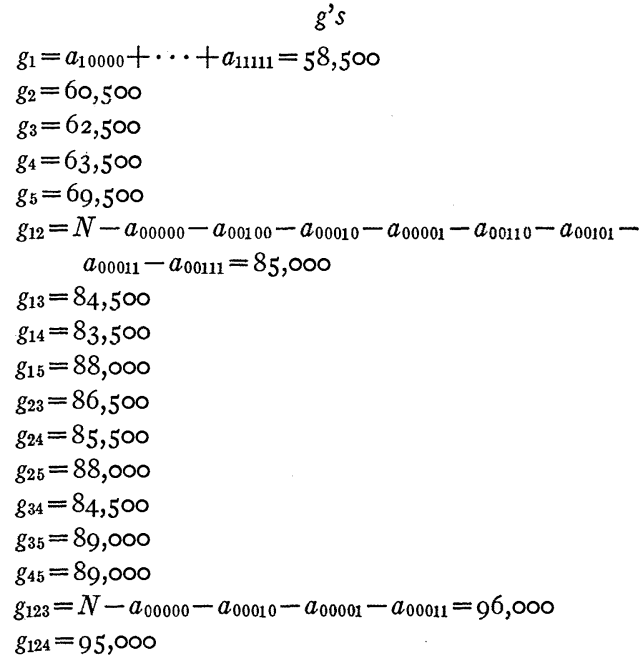

Sum of b's

$$
\begin{array}{r}
b_{1}=25,000 \\
b_{2}=5,000 \\
b_{3}=35,000 \\
b_{4}=32,500 \\
b_{5}=2,500
\end{array}
$$

$$
\begin{array}{r}
b_{1}+b_{2}=30,000 \\
b_{1}+b_{3}=60,000 \\
b_{1}+b_{4}=57,500 \\
b_{1}+b_{5}=27,500 \\
b_{2}+b_{3}=40,000 \\
b_{2}+b_{4}=37,500 \\
b_{2}+b_{5}=7,500 \\
b_{3}+b_{4}=67,500 \\
b_{2}+b_{5}=37,500 \\
b_{4}+b_{5}=35,000 \\
b_{1}+b_{2}+b_{3}=65,000 \\
b_{1}+b_{2}+b_{4}=62,500
\end{array}
$$




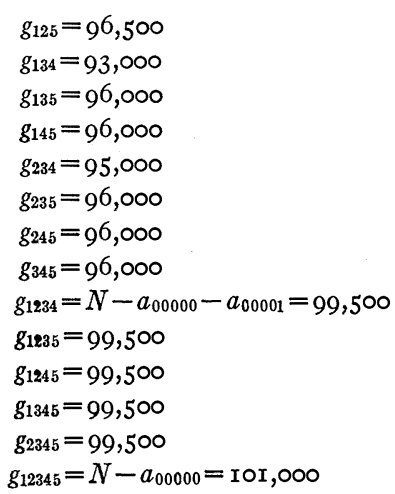

$$
\begin{aligned}
b_{1}+b_{2}+b_{5} & =32,500 \\
b_{1}+b_{3}+b_{4} & =92,500 \\
b_{1}+b_{3}+b_{5} & =62,500 \\
b_{1}+b_{4}+b_{5} & =60,000 \\
b_{2}+b_{3}+b_{4} & =72,500 \\
b_{2}+b_{3}+b_{5} & =42,500 \\
b_{2}+b_{4}+b_{5} & =40,000 \\
b_{3}+b_{4}+b_{5} & =70,000 \\
b_{1}+b_{2}+b_{3}+b_{4} & =97,500 \\
b_{1}+b_{2}+b_{3}+b_{5} & =67,500 \\
b_{1}+b_{2}+b_{4}+b_{5} & =65,000 \\
b_{1}+b_{3}+b_{4}+b_{5} & =95,000 \\
b_{2}+b_{3}+b_{4}+b_{5} & =75,000 \\
b_{1}+b_{2}+b_{3}+b_{4}+b_{5} & =100,000
\end{aligned}
$$

\begin{tabular}{|c|c|c|c|c|}
\hline 0 & 0 & 0 & 0 & 0 \\
\hline I 500 & 0 & 0 & 0 & 0 \\
\hline 0 & 1500 & 0 & 0 & 0 \\
\hline 0 & 0 & I 500 & 0 & 0 \\
\hline 0 & 0 & 0 & I 500 & 0 \\
\hline 0 & 0 & 0 & 0 & I 500 \\
\hline 2000 & 0 & 0 & 0 & 0 \\
\hline 2000 & 0 & 0 & 0 & 0 \\
\hline 2000 & 0 & 0 & 0 & 0 \\
\hline 3000 & 0 & 0 & 0 & 0 \\
\hline 0 & 500 & I 500 & 0 & 0 \\
\hline 0 & 0 & 0 & 2000 & 0 \\
\hline 0 & 3000 & 0 & 0 & 1000 \\
\hline 0 & 0 & I 500 & 0 & 0 \\
\hline 0 & 0 & 3000 & 0 & 0 \\
\hline 0 & 0 & 0 & 2000 & 0 \\
\hline I 500 & 0 & 0 & 0 & 0 \\
\hline 0 & 0 & 0 & I 500 & 0 \\
\hline 2000 & 0 & 0 & 0 & 0 \\
\hline 0 & 0 & 0 & 3000 & 0 \\
\hline 0 & 0 & 3000 & 0 & 0 \\
\hline 0 & 0 & 0 & 3000 & 0 \\
\hline 0 & 0 & 3000 & 0 & 0 \\
\hline 0 & 0 & 3000 & 0 & 0 \\
\hline 0 & 0 & 0 & 3000 & 0 \\
\hline 0 & 0 & 5000 & 0 & 0 \\
\hline 5000 & 0 & 0 & 0 & 0 \\
\hline 5000 & 0 & 0 & 0 & 0 \\
\hline 1000 & 0 & 0 & 6000 & 0 \\
\hline 0 & 0 & 7000 & 0 & 0 \\
\hline 0 & 0 & 6000 & 1000 & 0 \\
\hline 0 & 0 & 500 & 9500 & 0 \\
\hline
\end{tabular}

In every case the sum of $b$ 's is not greater than the corresponding $g$; therefore, the answer to part $(a)$ is yes.

The matrix $\left\|x_{i_{1} i_{2} \ldots i_{5} ; j}\right\|$ given below is a solution of part $(b)$ :

Personnel category

\footnotetext{
Quotas:

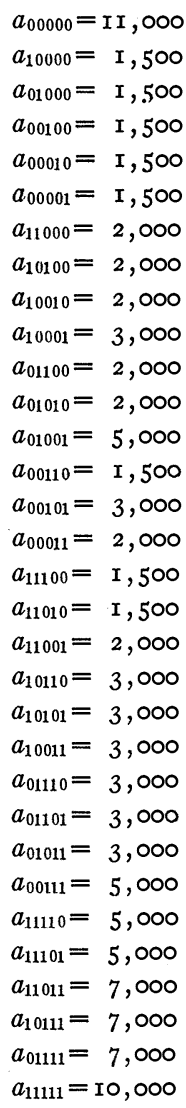


This matrix was obtained by carrying out the successive-reduction method. For convenience the rows were ordered as in (16), and values were assigned to all $x$ 's in one row before proceeding to the next. Setting $x_{01100 ; 2}$ and $x_{01100 ; 3}$ equal to 500 and 1500 , respectively, resulted in an equality of the sort mentioned in the description of the successive-reduction method. Specifically, the reduced $g_{134}$ was then equal to 77,500 and the sum of the reduced quotas of job categories 1,3 , and 4 was then 77,500 . Thus the total of all unassigned persons qualified in at least one of the job categories 1,3 , and 4 was then just equal to the total number of persons required in those job categories. At this stage the original problem could be 'factored' into two smaller problems - one of them having three job categories (namely 1,3 , and 4) and the other having two job categories (namely 2 and 5). Accordingly, we note that below the row of $a_{01100}$ in (16) the only persons assigned to job categories 2 and 5 are those unqualified in each of 1,3 , and 4 .

About three hours of hand calculation was required to determine the existence of a solution and find the solution in (16).

\section{REFERENCES}

1. C. W. Churchman, R. L. Ackoff, And E. L. Arnoff, Introduction to Operations Research, Wiley, New York, 1957.

2. G. B. Dantzig, "Application of the Simplex Method to a Transportation Problem," Chapter XXIII in Activity Analysis of Production and Allocation (T. C. Koopmans, Ed.), Wiley, New York, 1951.

3. J. A. Joseph, The Application of Linear Programming to Weapon Selection and Target Analysis, Operations Analysis Technical Memorandum No. 42, Operations Analysis Division, Directorate of Operations, DCS/O, Headquarters United States Air Force, Washington 25, D. C., 5 January 1954.

4. D. F. Votaw, JR., ANd J. T. DAILEY, Assignment of Personnel to Jobs, Research Bulletin 52-24, Human Resources Research Center, Lackland Air Force Base, San Antonio, Texas, August 1952.

5. ——, "Methods of Solving Some Personnel-Classification Problems," Psychometrika 17, 255-266 (1952). 